width $=.75$

Electronic Supplementary Material

\title{
Vibrational entropy stabilizes distorted half-Heusler structures
}

Shuping Guo, ${ }^{a, b, c}$ Shashwat Anand, ${ }^{b}$ Yongsheng Zhang ${ }^{* a, c}$ and G. Jeffrey Snyder*b

\footnotetext{
${ }^{a}$ Key Laboratory of Materials Physics, Institute of Solid State Physics, Chinese Academy of Sciences, Hefei 230031, China.

${ }^{b}$ Department of Materials Science and Engineering, Northwestern University, Evanston, IL 60208, USA

${ }^{c}$ Science Island Branch of Graduate School, University of Science and Technology of China, Hefei 230026, China.

*E-mail: yshzhang@theory.issp.ac.cn, jeff.snyder@northwestern.edu
} 
Table S1: The correlation function $\overline{\prod_{f}}$ of first $(2,1)$, second $(2,2)$, third $(2,3)$ as well as forth $(2,4)$ pair and first $(3,1)$ as well as second $(3,2)$ triple interactions of orthorhombic and cubic $\mathrm{Ti}_{0.5} \mathrm{Hf}_{0.5} \mathrm{NiGe}$ SQS structures, respectively.

\begin{tabular}{ccccccc}
\hline \hline Compound & $\overline{\prod_{2,1}}$ & $\overline{\prod_{2,2}}$ & $\overline{\prod_{2,3}}$ & $\overline{\prod_{2,4}}$ & $\overline{\prod_{3,1}}$ & $\overline{\prod_{3,2}}$ \\
\hline $\mathrm{Ti}_{0.5} \mathrm{Hf}_{0.5}$ NiGe-pnma & 0 & 0 & 0 & 0 & 0 & 0 \\
$\mathrm{Ti}_{0.5} \mathrm{Hf}_{0.5}$ NiGe-cubic & 0 & 0 & 0 & 0 & 0 & 0 \\
\hline \hline
\end{tabular}

Table S2: Vibrational entropy $\left(S_{v i b}, \mathrm{~J} / \mathrm{K} / \mathrm{mol}\right)$ and heat capacity $\left(C_{v}, \mathrm{~J} / \mathrm{K} / \mathrm{mol}\right)$ at $1000 \mathrm{~K}$ for orthorhombic (Pnma) and cubic $(F \overline{4} 3 \mathrm{~m})$ phases of 16 half-Heusler systems.

\begin{tabular}{ccccc}
\hline \hline Compound & $S_{v i b-p n m a}$ & $S_{v i b-h h}$ & $C_{v-p n m a}$ & $C_{v-h h}$ \\
& $\mathrm{~J} / \mathrm{K} / \mathrm{mol}$ & $\mathrm{J} / \mathrm{K} / \mathrm{mol}$ & $\mathrm{J} / \mathrm{K} / \mathrm{mol}$ & $\mathrm{J} / \mathrm{K} / \mathrm{mol}$ \\
\hline TiNiGe & 683.88 & 165.22 & 296.81 & 74.06 \\
HfNiGe & 749.78 & 181.01 & 297.56 & 74.26 \\
HfCoAs & 735.76 & 179.33 & 297.41 & 74.24 \\
ZrPtGe & 777.41 & 189.98 & 297.78 & 74.35 \\
TiRhAs & 715.60 & 174.14 & 297.21 & 74.17 \\
TaRuAs & 753.56 & 176.94 & 296.64 & 73.45 \\
ZrIrAs & 771.93 & 187.92 & 297.46 & 74.07 \\
VCoGe & 672.85 & 161.98 & 296.49 & 73.98 \\
NbCoGe & 691.71 & 167.86 & 296.88 & 74.10 \\
TaCoGe & 718.20 & 172.85 & 297.04 & 74.14 \\
TiCoAs & 666.83 & 162.94 & 296.57 & 74.02 \\
NbFeAs & 685.32 & 164.98 & 296.74 & 74.04 \\
TaFeAs & 715.33 & 171.28 & 296.95 & 74.10 \\
NbIrGe & 751.51 & 181.51 & 297.50 & 74.27 \\
TiPtGe & 754.96 & 181.53 & 297.48 & 74.23 \\
VRuAs & 719.91 & 171.58 & 297.16 & 74.12 \\
\hline \hline
\end{tabular}



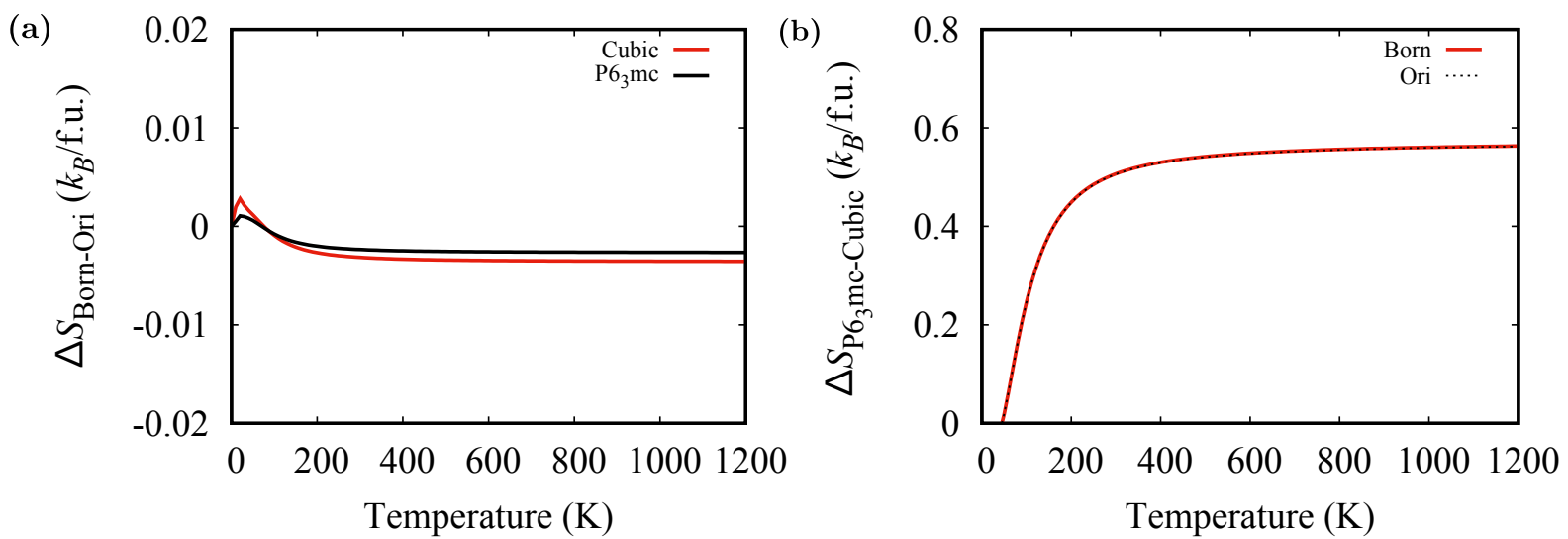

Fig. S1: Temperature dependence of vibrational entropy difference (a) between with and without considering dipole-dipole interactions and (b) between hexagonal $\left(P 6{ }_{3} m c\right)$ and cubic $(F \overline{4} 3 m)$ for LiZnSb, respectively. 


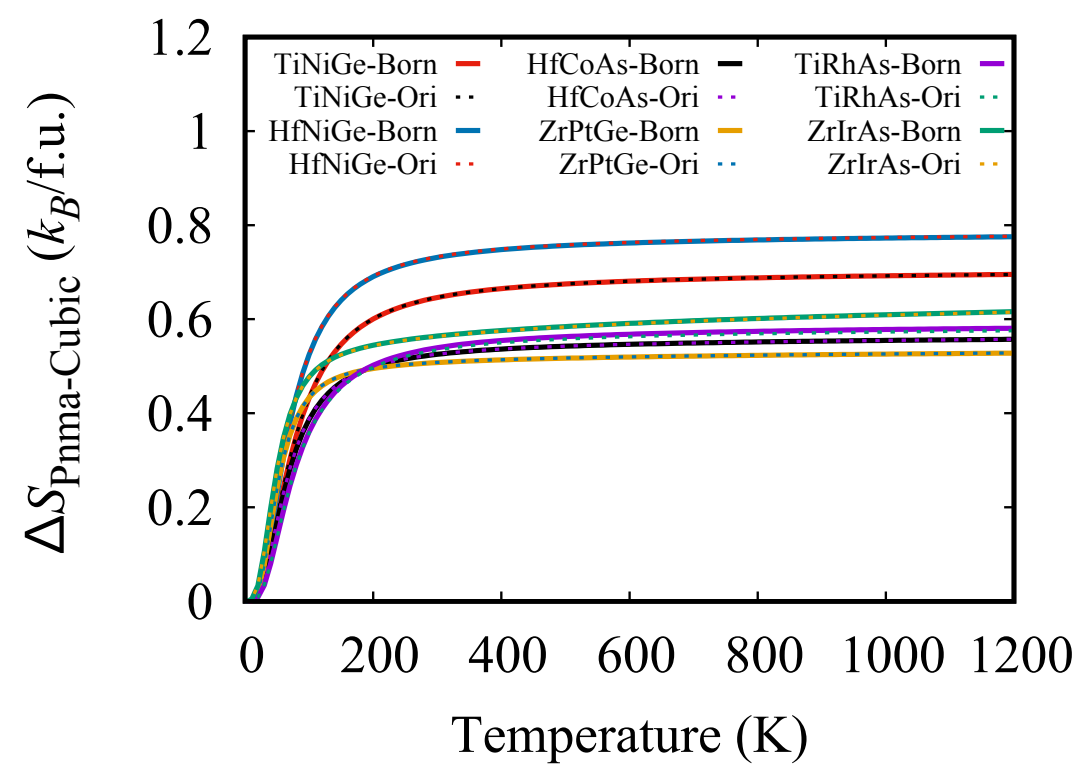

Fig. S2: Temperature dependence of vibrational entropy difference between of orthorhombic $(P n m a)$ and cubic $(F \overline{4} 3 m)$ phases for six half-Heusler systems with and without dipole-dipole interactions. 

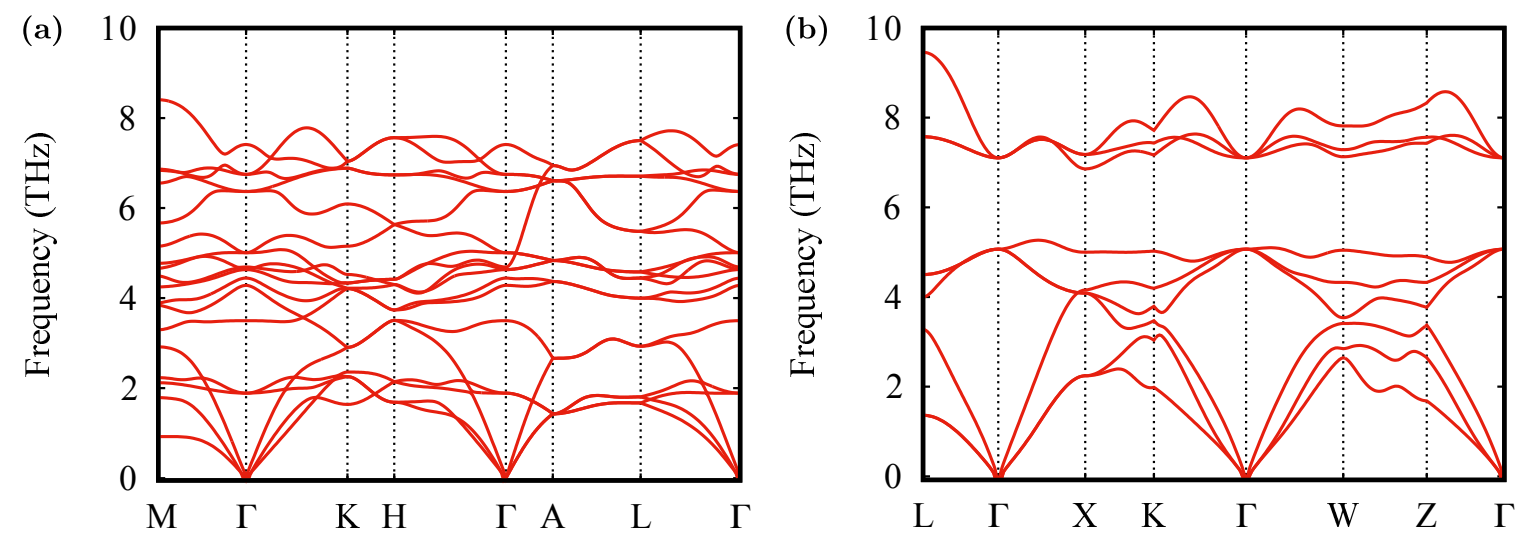

Fig. S3: The phonon disperisions of (a) hexagonal $\left(P 6_{3} m c\right)$ and (b) cubic $(F \overline{4} 3 m)$ phases of LiZnSb, respectively. 

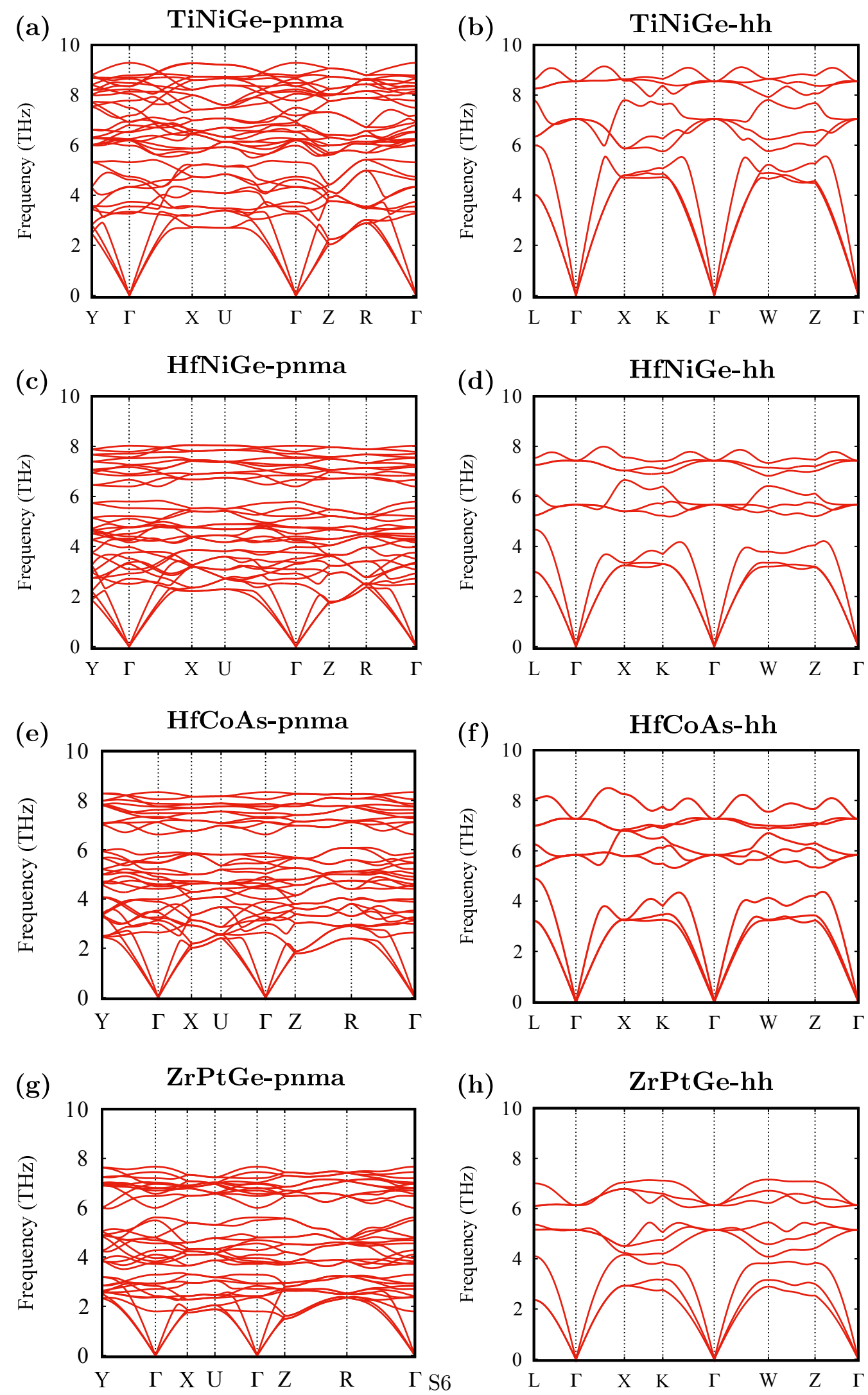

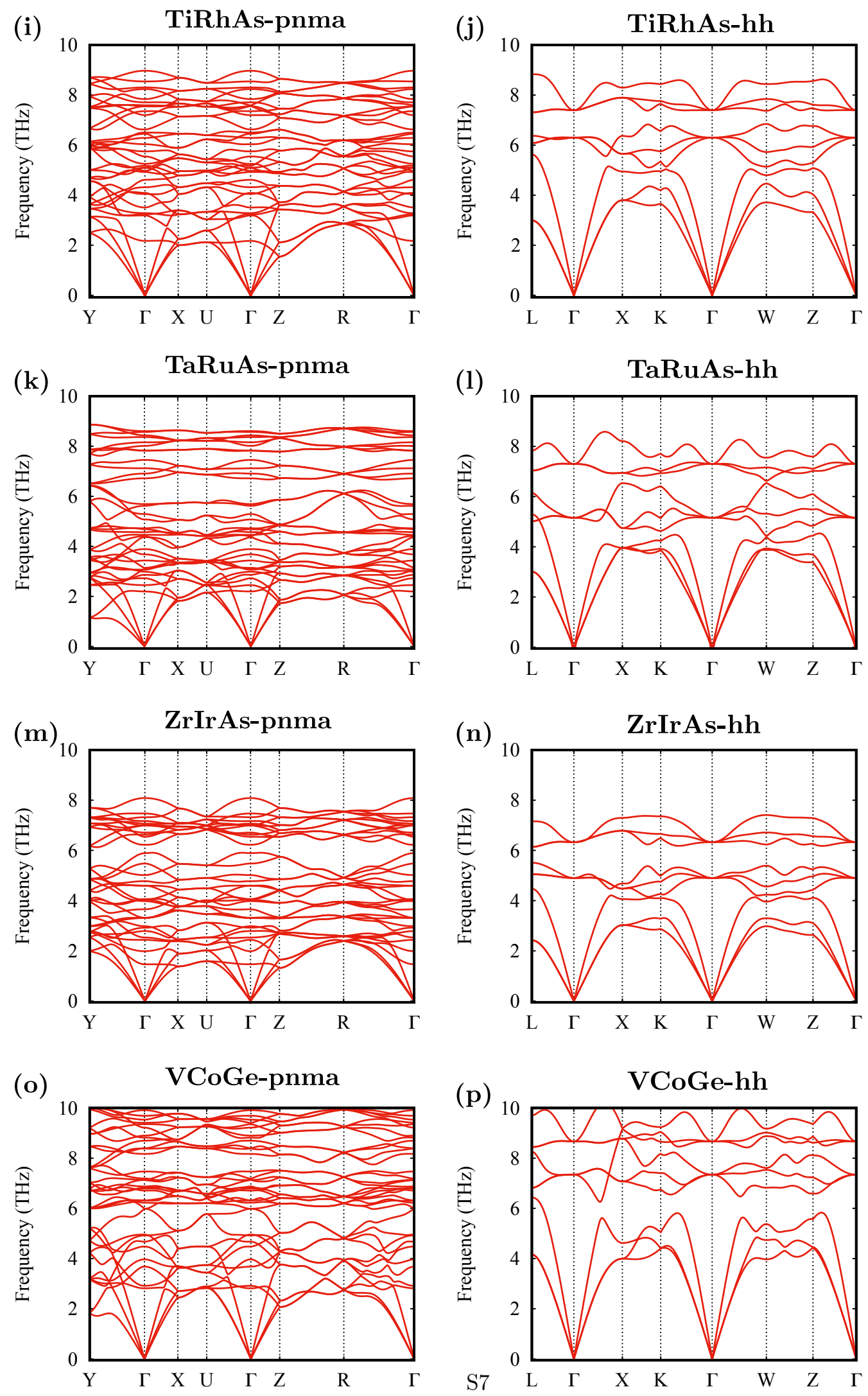

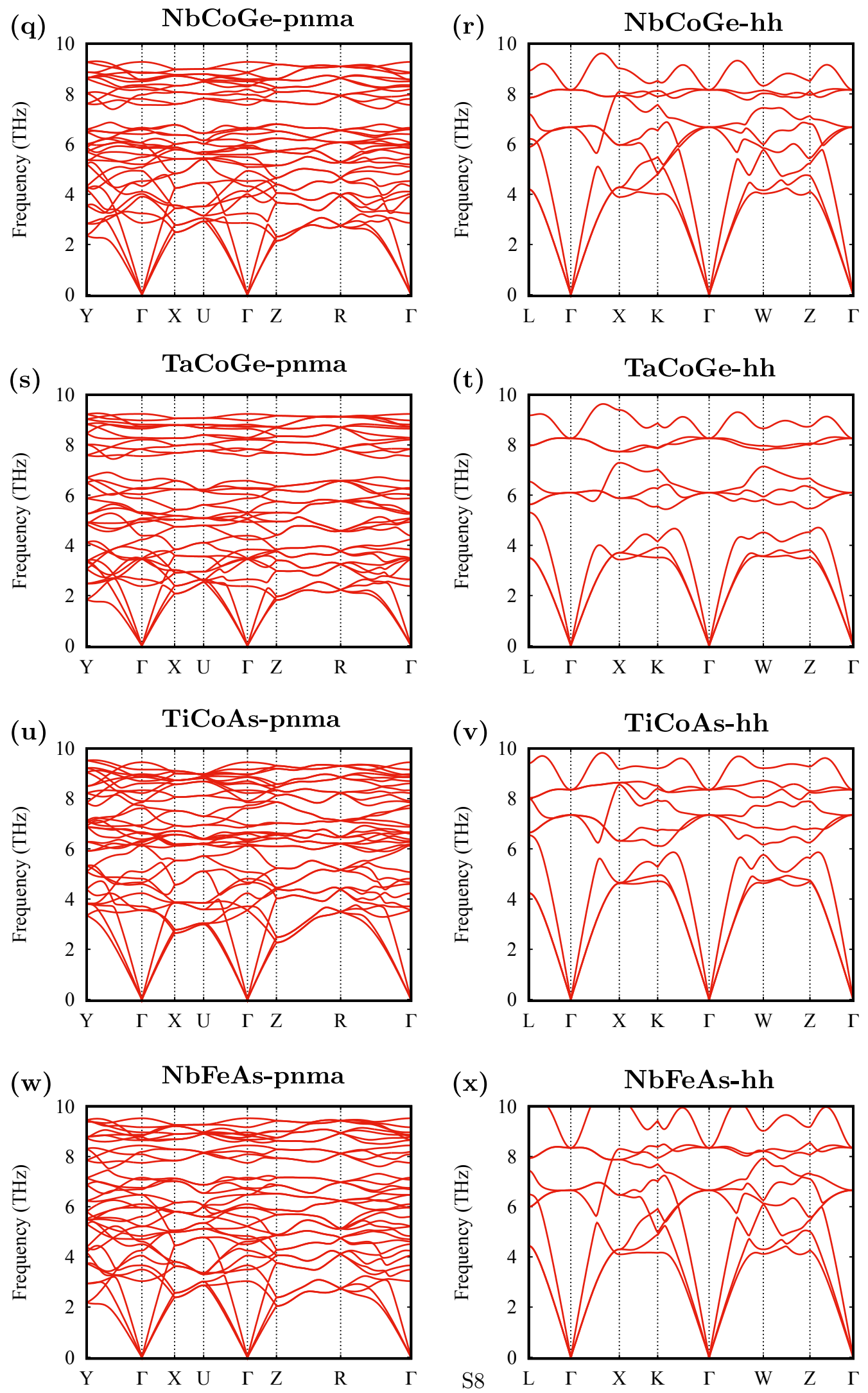

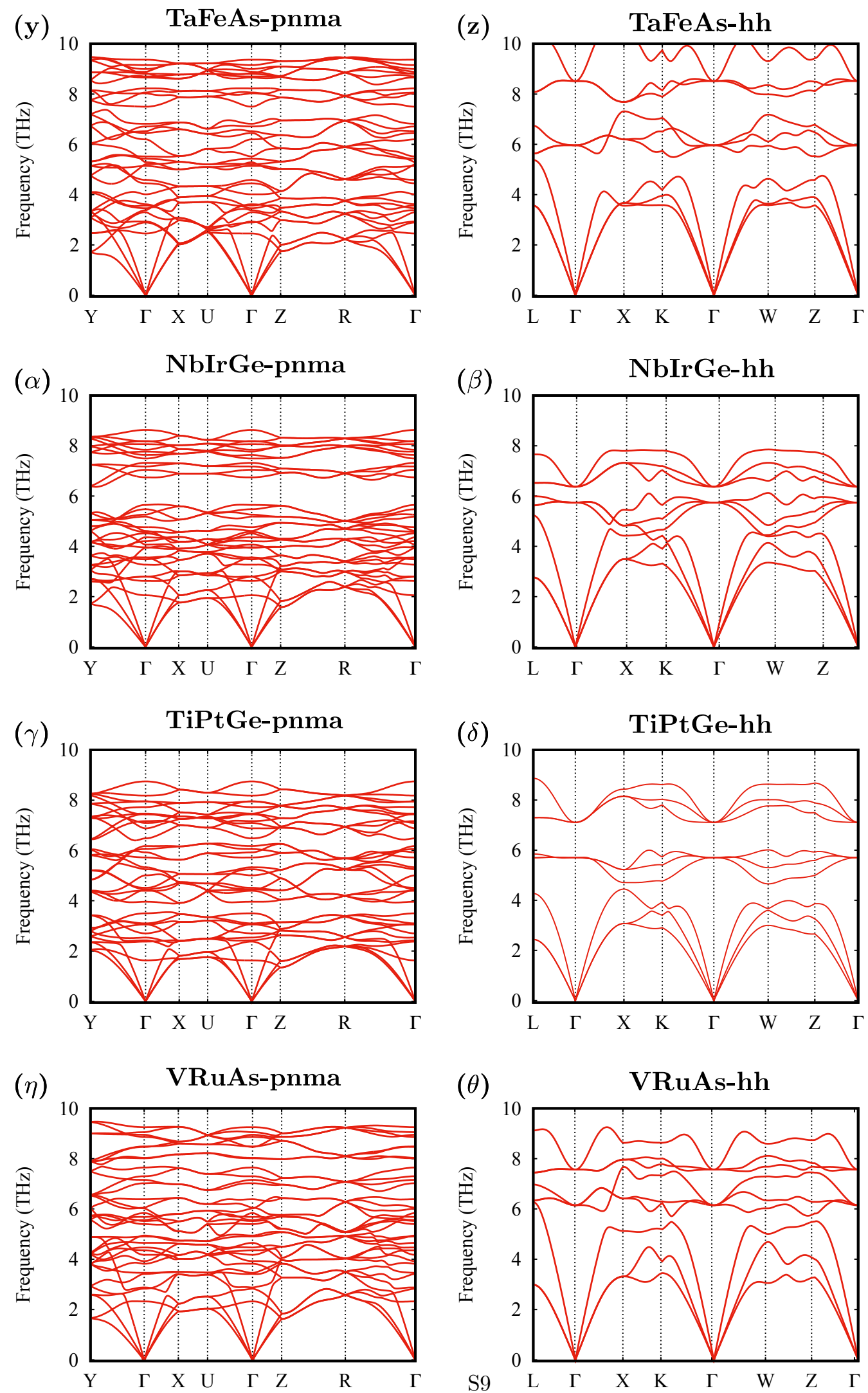

Fig. S4: The phonon dispersions of orthorhombic (Pnma) and Cubic $(F \overline{4} 3 m)$ phases for 16 systems, respectively. 

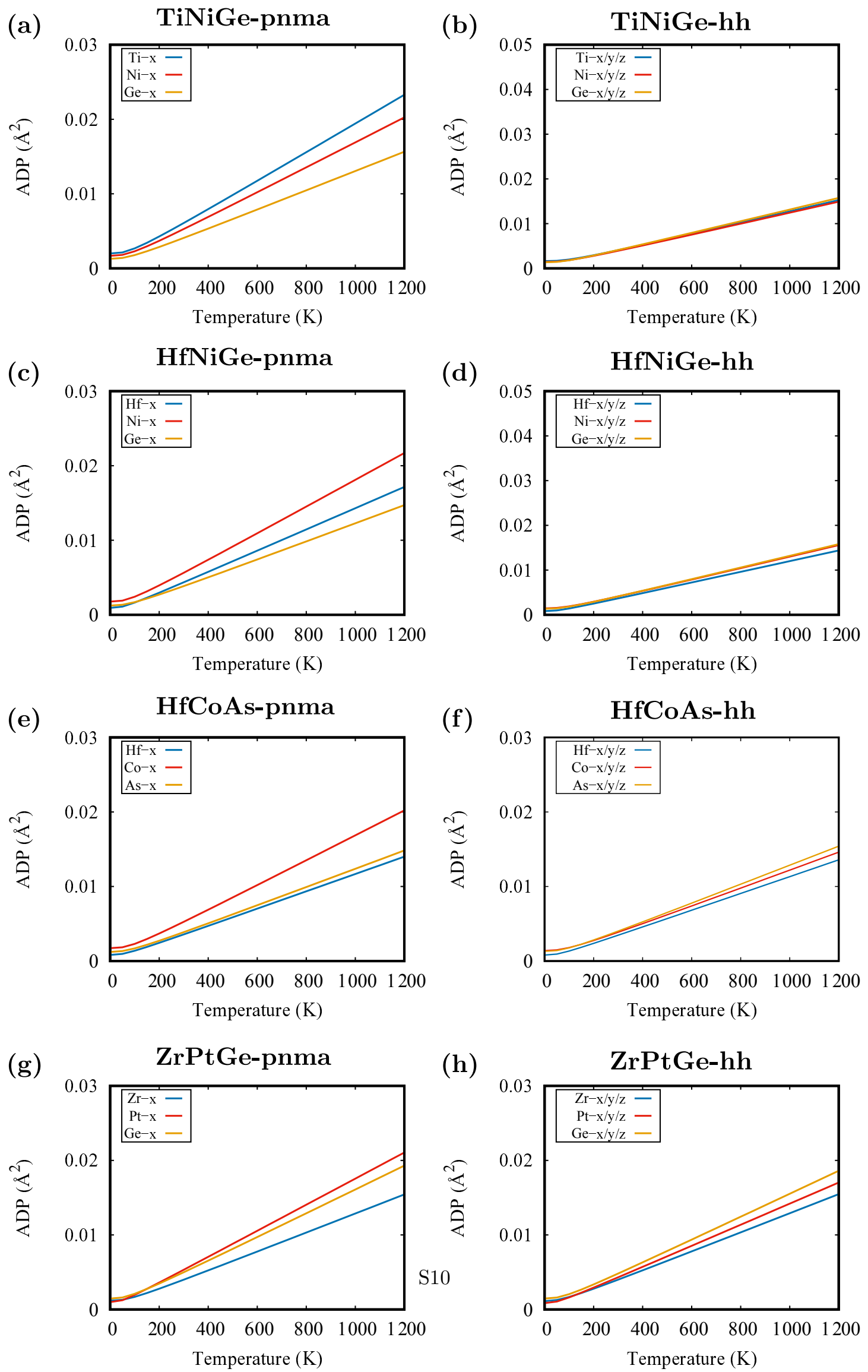

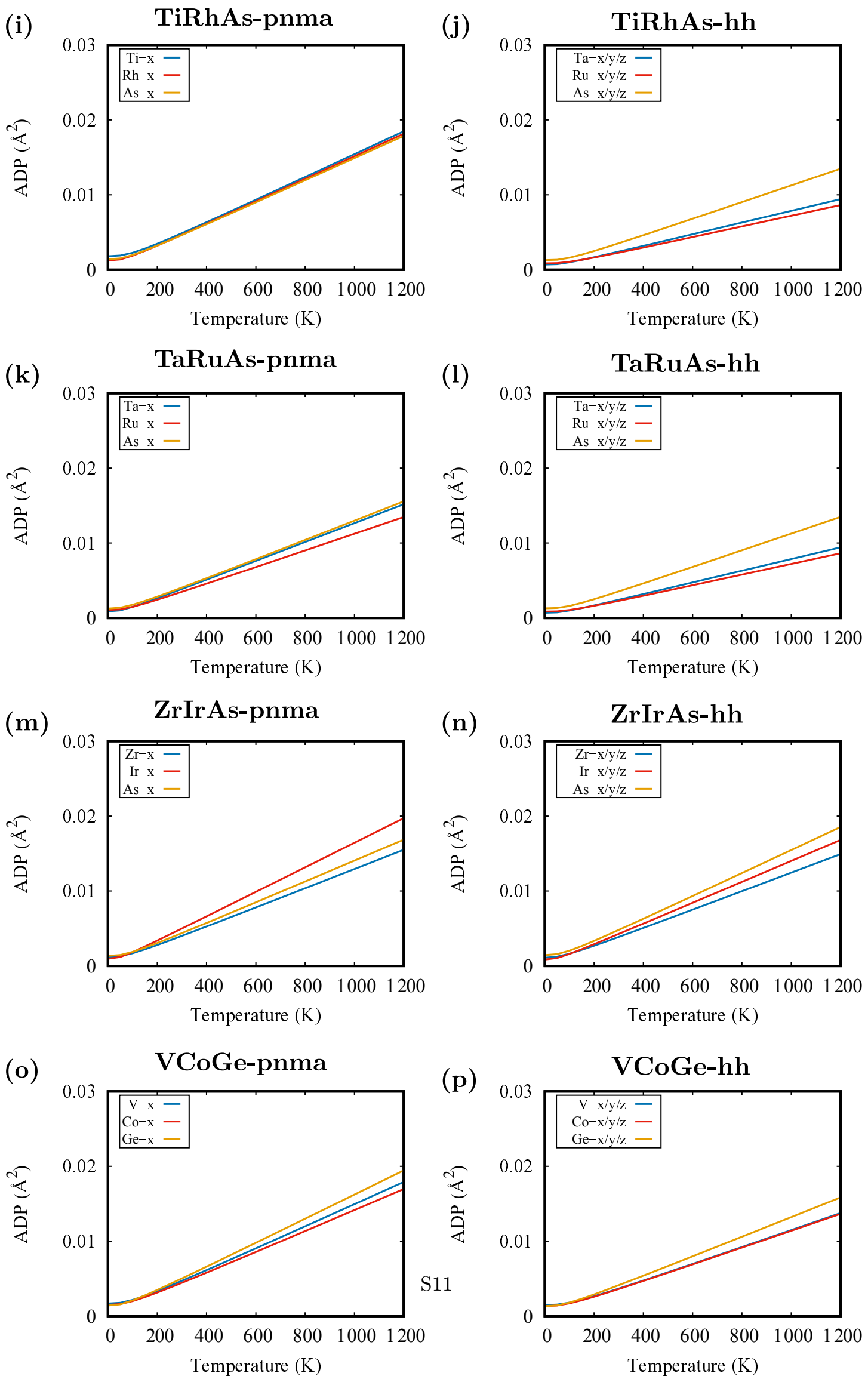

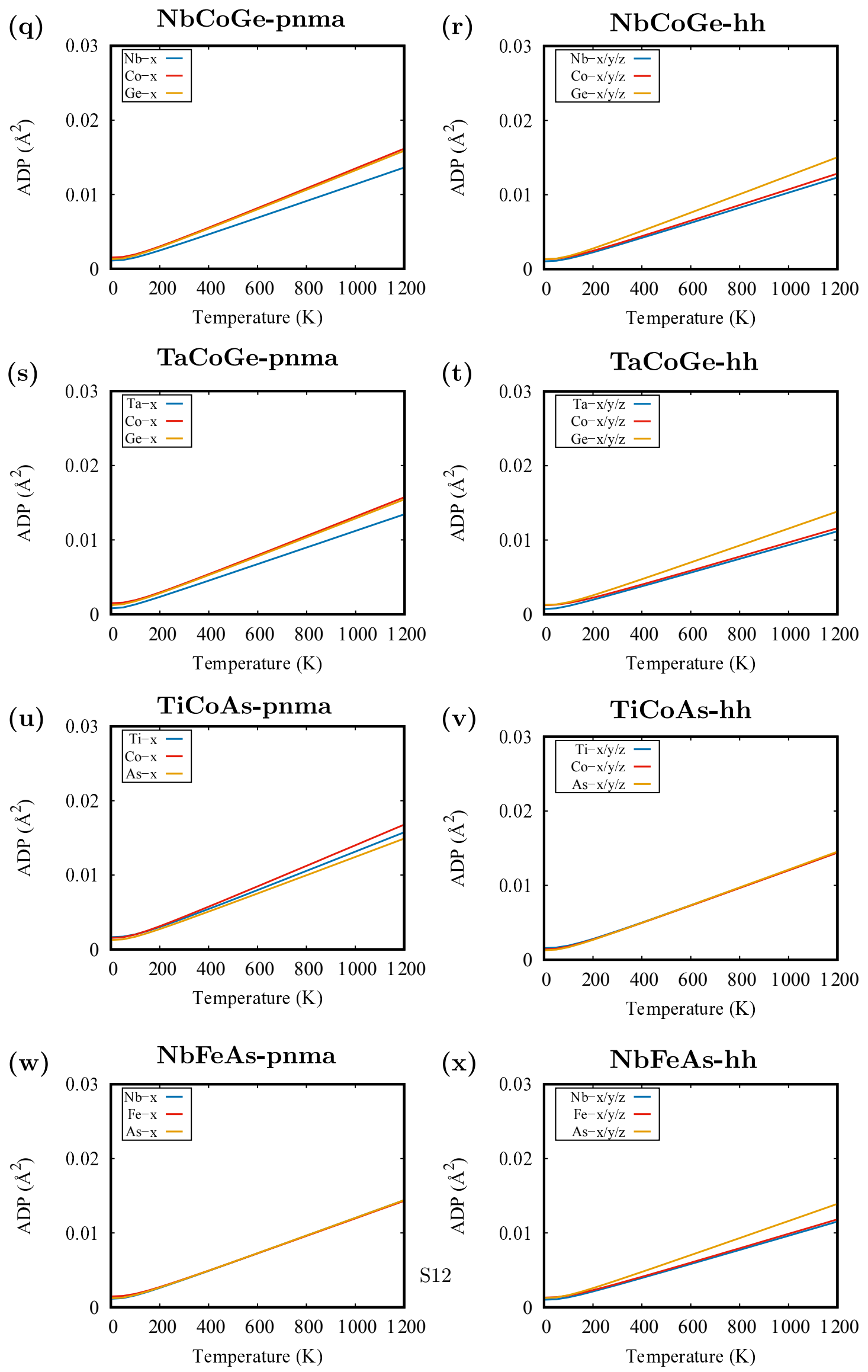

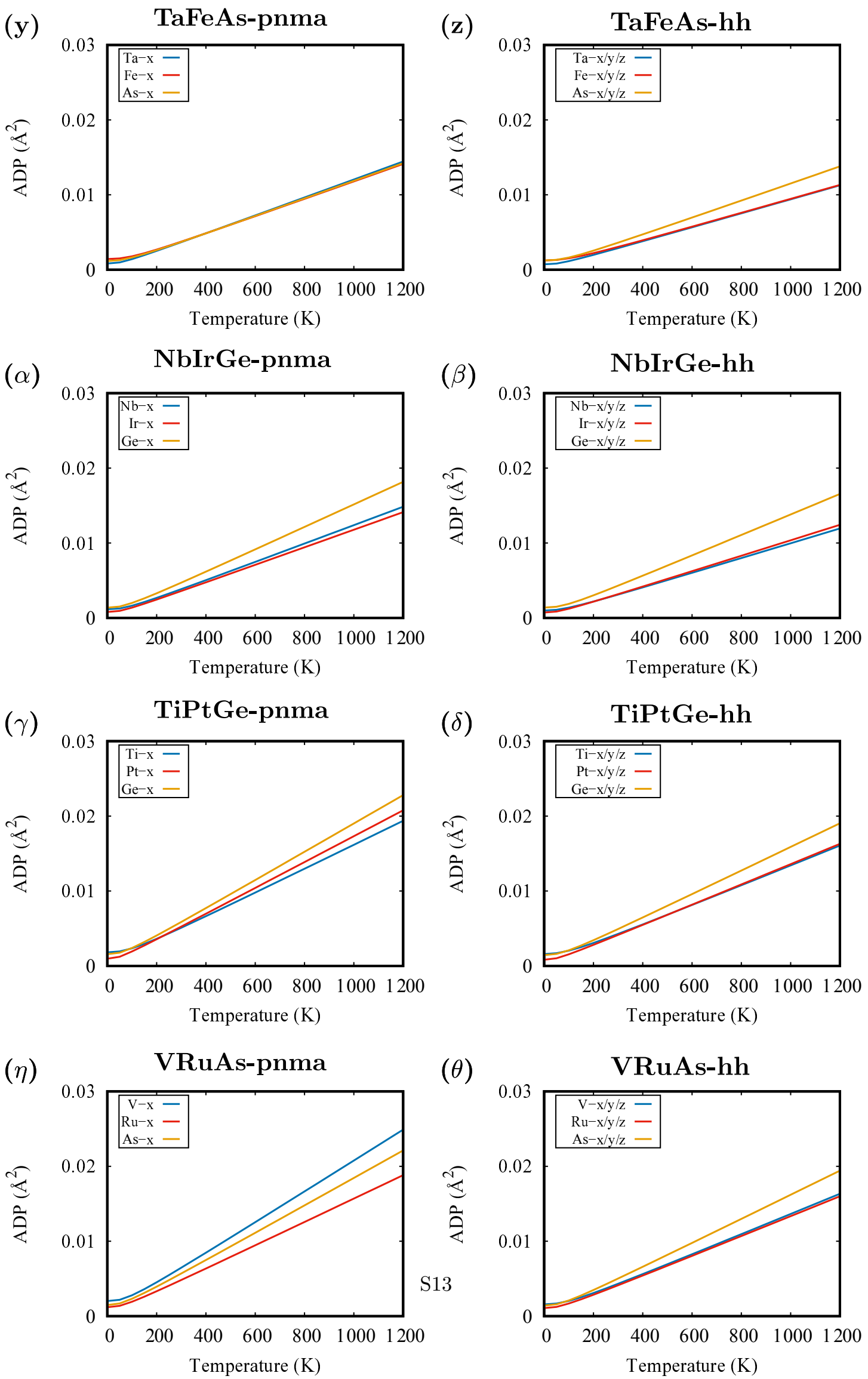

Fig. S5: The atomic displacement parameter along $x$ direction of of orthorhombic (Pnma) and Cubic $(F \overline{4} 3 m)$ phases for 16 systems. The blue, red and yellow lines represnt A, B and X atoms, respectively. Since cubic phase is isotropic, the ADPs are the same along $x, y$ and $z$ directions. 

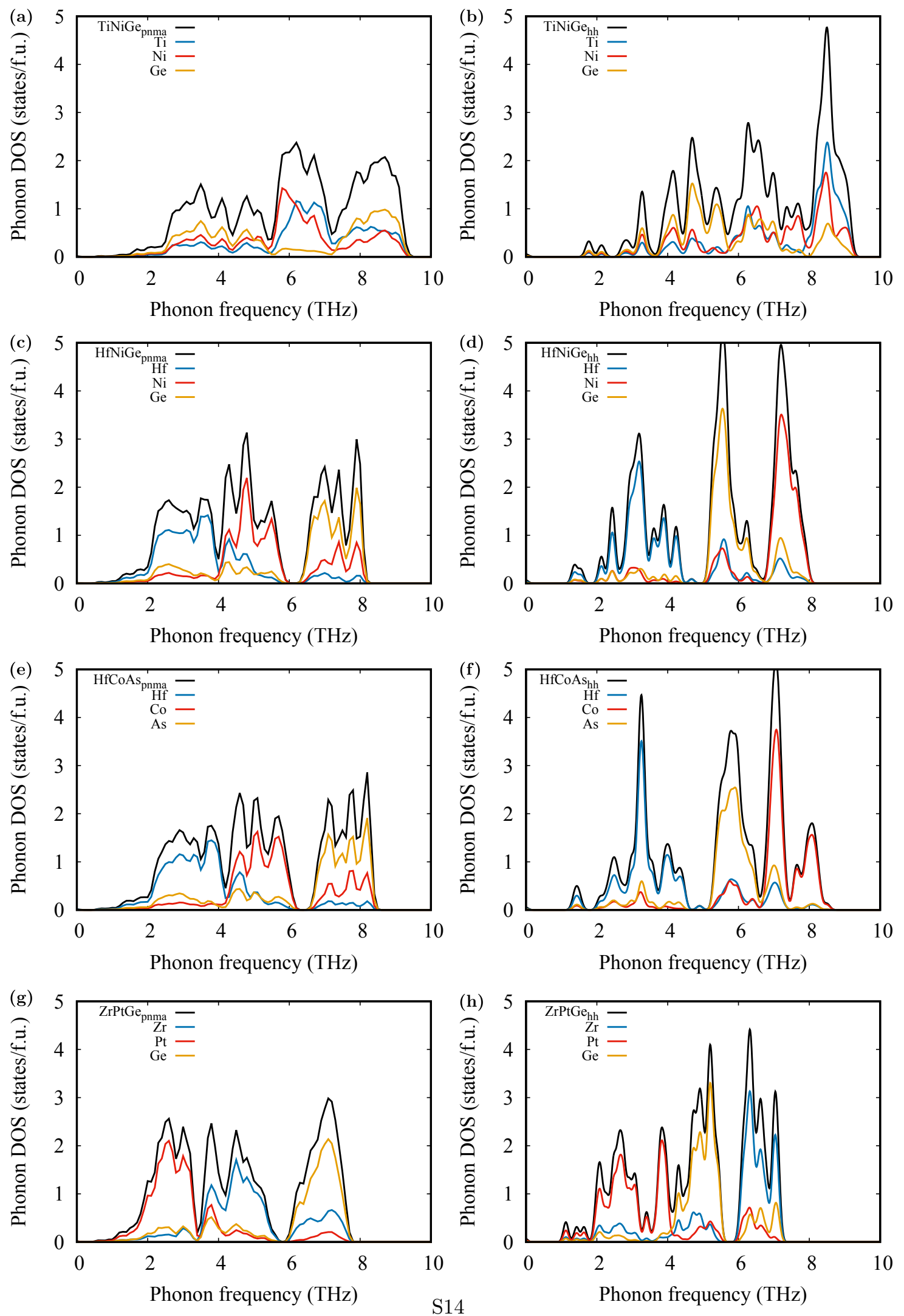

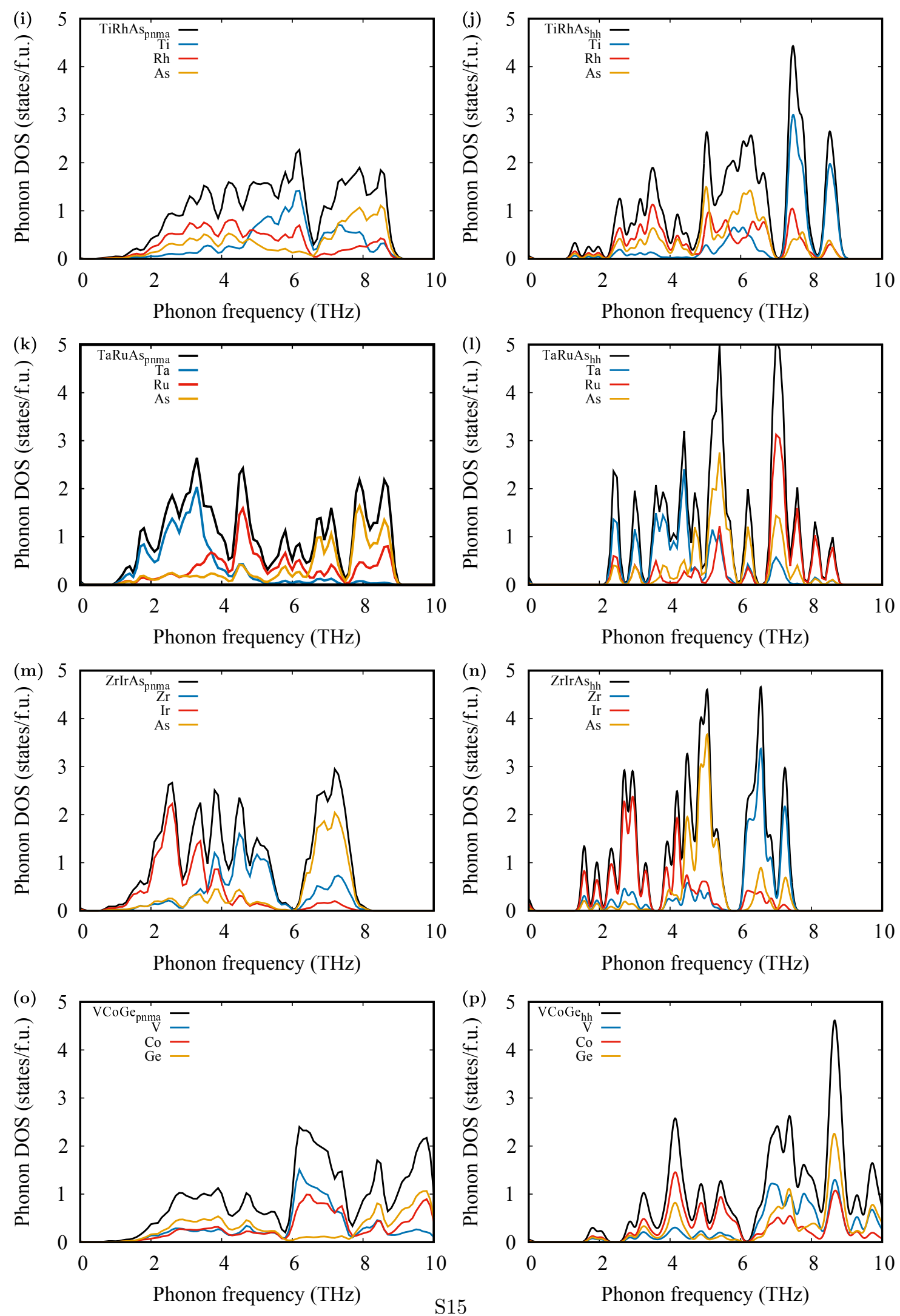

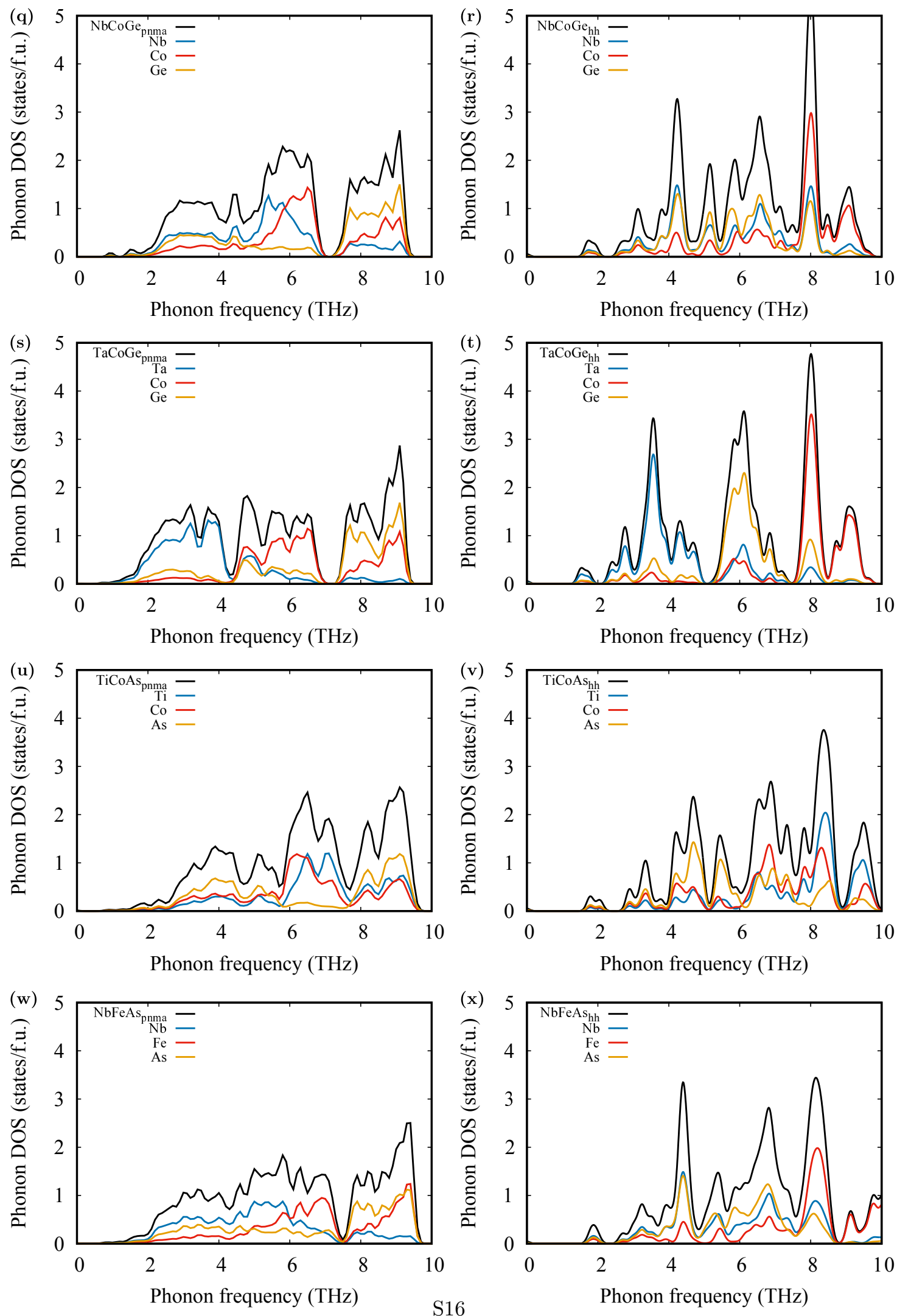

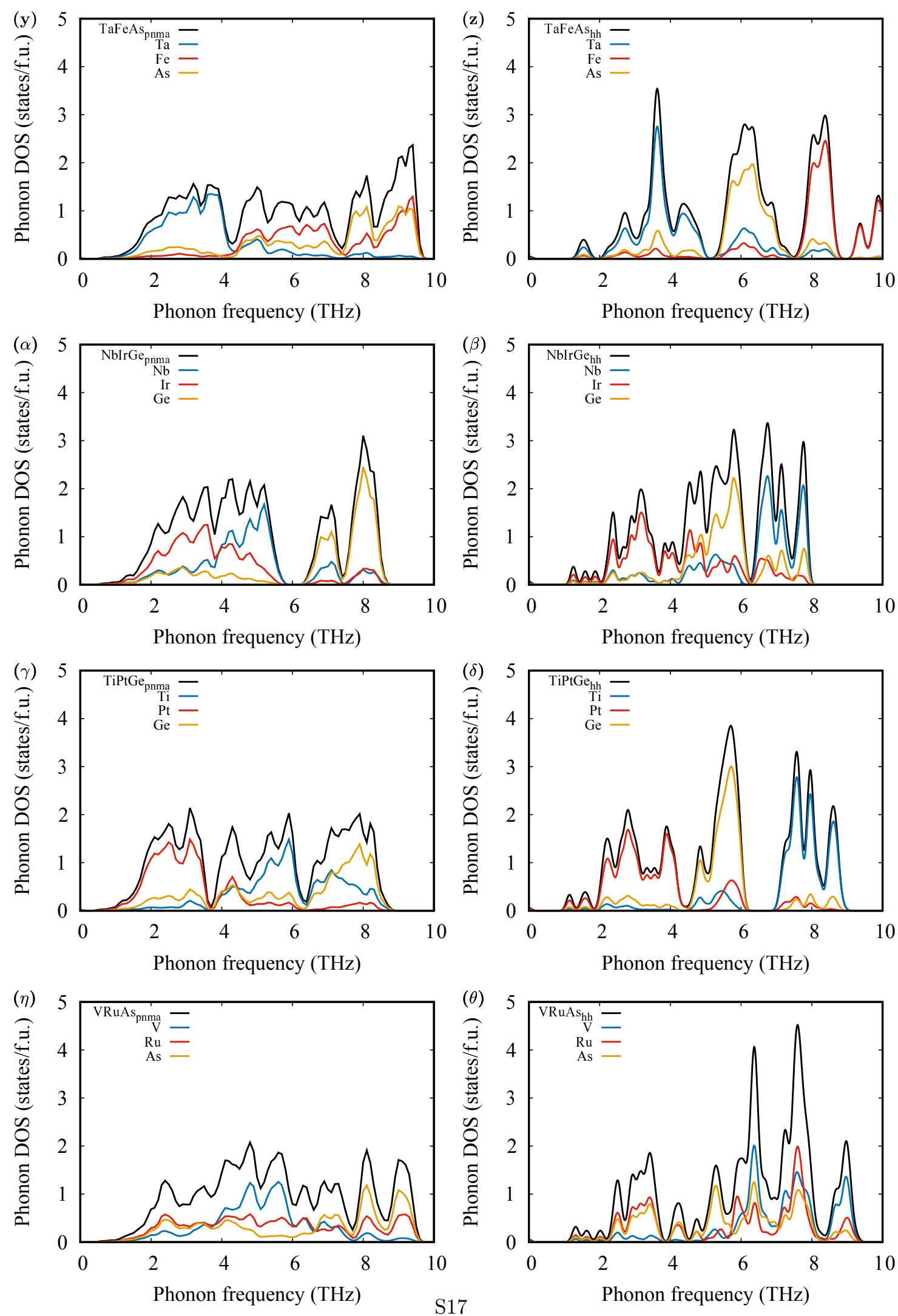

Fig. S6: The phonon DOS of orthorhombic $($ Pnma) and Cubic $(F \overline{4} 3 m)$ phases for 16 systems, the black, blue, red and yellow lines represent the total and partial (A, B and X atoms) DOS, respectively. 